\title{
SUPERVISORY FAULT TOLERANT CONTROL WITH INTEGRATED FAULT DETECTION AND ISOLATION: A SWITCHED SYSTEM APPROACH
}

\author{
HAO YANG *, BIN JIANG *, VINCENT COCQUEMPOT **, LINGLI LU * \\ * College of Automation Engineering \\ Nanjing University of Aeronautics and Astronautics, 29 YuDao Street, Nanjing, China \\ e-mail: \{haoyang, binjiang\}@nuaa.edu.cn, linglanzhishui@163.com \\ ** LAGIS Laboratory, UMR CNRS 8219 \\ Lille 1 University: Sciences and Technologies, 59655 Villeneuve d'Ascq, France \\ e-mail: vincent.cocquempot@univ-lille1.fr
}

\begin{abstract}
This paper focuses on supervisory fault tolerant control design for a class of systems with faults ranging over a finite cover. The proposed framework is based on a switched system approach, and relies on a supervisory switching within a family of pre-computed candidate controllers without individual fault detection and isolation schemes. Each fault set can be accommodated either by one candidate controller or by a set of controllers under an appropriate switching law. Two aircraft examples are included to illustrate the efficiency of the proposed method.
\end{abstract}

Keywords: fault tolerant control, fault detection and isolation, switching control, switched systems.

\section{Introduction}

Fault Detection and Isolation (FDI) and Fault Tolerant Control (FTC) are aimed at guaranteeing the primary system goal to be achieved in spite of faults (Patton et al., 2000; Blanke et al., 2006; Zhang and Jiang, 2008; Yang et al., 2010). The potential faults in a complex system often range over a very large region. A single controller (even an adaptive one) is often hard to design to stabilize all faulty situations effectively. General supervisory FTC approaches assume that the plant model belongs to a pre-specified set of models, including the nominal situation and all possible faulty situations, and that there exists a finite family of candidate controllers such that the faulty system is stabilized when controlled by at least one of those candidate controllers (Staroswiecki and Gehin, 2001; Parisini and Sacone, 2001).

The classical supervisory FTC approach, as shown by Fig. 1, follows three steps: (1) detect the occurrence of a fault; (2) identify the current fault situation; (3) switch to the related controller. There are three limitations behind such a framework:

L1. An individual fault detection scheme is required, which often relies on a set of residuals. It is well known that an unappropriate residual may lead to a false alarm or a missed detection (Patton et al., 2000). This also introduces a detection delay during which the faulty system is controlled by the original controller, the stability may be violated, or some unexpected behaviors may appear.

L2. A bank of filters/models has to be designed and work in parallel with the plant to identify the current fault (Zhang et al., 2008). This makes the FTC system complicated. An identification delay exists during which the faulty system is still controlled by the original controller. Stability may also be violated. Moreover, designing these filters often requires some structure conditions on the plant. The actual fault may be ill-isolated, possibly leading to fatal consequences.

L3. Each possible fault set can be accommodated by at least one of the candidate controllers. However, some complex faults are often difficult to be accommodated by only one controller.

In this work, we propose a new supervisory FTC scheme as shown in Fig. 2, where FDI and FTC are integrated via a switching algorithm. Controllers are sequentially switched until the appropriate one is found, and the 


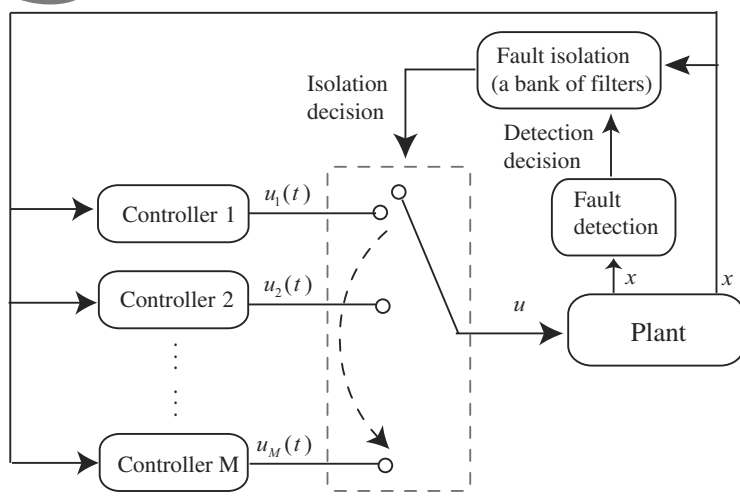

Fig. 1. Classical supervisory FTC framework.

fault isolation boils down to finding the correct controller, which can be directly applied once selected. The switching delay in setting the correct controller still exists, but there is no individual detection and isolation algorithm, which makes the scheme simpler and more easily verifiable. Moreover, the switching delay can be controlled according to the design parameters, while the state remains bounded during this delay as will be shown.

The proposed approach relaxes L1-L3 and has two good features:

1. FDI and FTC are integrated via a control switching algorithm. Individual detection and isolation schemes are not needed. Thus, the delay of detection and isolation is avoided. A switching delay exists during which the system remains stable.

2. Each faulty system is allowed not to be stabilized by one individual controller but can be stabilized by a set of controllers under an appropriate switching law.

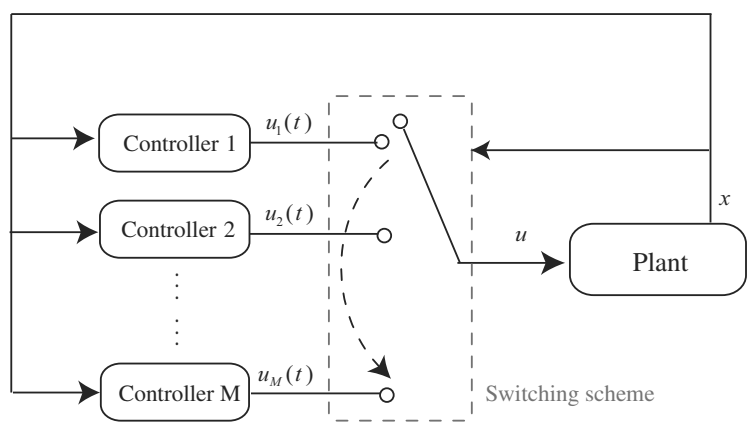

Fig. 2. New supervisory FTC framework.

The key condition of realizing such an integrated FDI/FTC scheme is to guarantee the stability of the system in the pre-fault period, the FDI/FTC period and the postFTC period by controller switching. In fact, a system under switching within a family of pre-computed candidate controllers can be described naturally by a switched system, since each mode of the switched system could represent one of the control configurations, while a switching from one configuration to another one is described using a switching function. Consequently, the system stability during the switching period of controllers is equivalent to the stability of the switched system. Our proposed supervisory FTC framework is based on stability criteria of the switched system with unstable modes.

The remainder of the paper is organized as follows. Section 2 gives some preliminaries. Section 3 discusses supervisory FTC with relaxation of L1-L2, while Section 4 focuses on the relaxation of L1-L3, followed by some concluding remarks in Section 5.

\section{Preliminaries}

In the following, let $\mathbb{R}$ denote the field of real numbers, $\mathbb{R}^{r}$ the $r$-dimensional real vector space and $|\cdot|$ the Euclidean norm. Class $\mathcal{K}$ is a class of strictly increasing and continuous functions $[0, \infty) \rightarrow[0, \infty)$ which are zero at zero. Class $\mathcal{K}_{\infty}$ is the subset of $\mathcal{K}$ consisting of all those functions that are unbounded. Furthermore, $\beta:[0, \infty) \times[0, \infty) \rightarrow[0, \infty)$ belongs to the class $\mathcal{K} \mathcal{L}$ if $\beta(\cdot, t)$ is of class $\mathcal{K}$ for each fixed $t \geq 0$ and $\beta(s, t)$ decreases to 0 as $t \rightarrow \infty$ for each fixed $s \geq 0$. Moreover, $t^{-}$ denotes the left limit time instant of $t$. Finally, $(\cdot)^{\top}$ is the transposition.

The system considered takes the general nonlinear form

$$
\dot{x}(t)=G(x(t), u(t), f(u(t), x(t)))
$$

with measurable states $x \in \mathbb{R}^{n}$ and inputs $u \in \mathbb{R}^{p}$. $G$ is smooth. Process and/or actuator faults are represented by the function $f: \mathbb{R}^{p} \times \mathbb{R}^{n} \rightarrow \mathcal{F}$, where $\mathcal{F} \subset \bigcup_{i \in \mathcal{N}=\{1, \ldots, N\}} \mathcal{F}_{i} \subset \mathbb{R}^{q}$ and $\mathcal{F}_{i}$ is the $i$-th set of fault vectors, $N$ is a finite number, the fault free operation is $\mathcal{F}_{N}=\{0\}$.

The property that we wish to be invariant under the faults in $\mathcal{F}$ is that the system (1) remains stable whatever the fault $i \in \mathcal{N}$ and whenever it occurs, i.e., for any $\epsilon>0$, there exists a $\delta>0$ such that $|x(t)| \leq \epsilon, t \geq 0$, whenever $|x(0)| \leq \delta$.

Suppose that there are $\omega$ pre-computed candidate controllers for the supervision purpose and $\omega>0$ is a finite number. Define $\Omega \triangleq\{1,2, \ldots, \omega\}$. Write $u_{i}(t)$ for the signal the $i$-th controller, $i \in \Omega$.

The system (1) under controller switching among $u_{i}(t), i \in \Omega$, can be rewritten as the following switched system:

$$
\dot{x}(t)=G\left(x(t), u_{\sigma(t)}(t), f\left(u_{\sigma(t)}(t), x(t)\right)\right),
$$

where $\sigma(t):[0, \infty) \rightarrow \Omega$ denotes the switching function, which is assumed to be piecewise constant and continuous 
from the right. It is clear that the original system under one of the controllers can be regarded as one of the modes of the switched system.

\section{Supervisory FTC: Relaxation of L1-L2}

For the sake of simplicity, it is assumed in this section that $\Omega=\mathcal{N}$, i.e., each control law $u_{i}$ is associated with a fault $i, i \in \mathcal{N}$

Assumption 1. For any $i, j \in \mathcal{N}$, there exists a family of continuous non-negative functions $V_{i}(x): \mathbb{R}^{n} \rightarrow \mathbb{R}_{\geq 0}$, and functions $\alpha_{1}, \alpha_{2} \in \mathcal{K}_{\infty}, \lambda_{0}, \lambda_{1}>0, \mu_{0} \geq 1$ such that

$$
\begin{aligned}
& \alpha_{1}(|x|) \leq V_{i}(x) \leq \alpha_{2}(|x|), \\
& u=u_{i}, f \in \mathcal{F}_{i} \Longrightarrow \dot{V}_{i}(x) \leq-\lambda_{0} V_{i}(x), \\
& u=u_{i}, f \in \mathcal{F}_{j}, j \neq i \Longrightarrow \dot{V}_{i}(x) \leq \lambda_{1} V_{i}(x), \\
& V_{i}(x) \leq \mu_{0} V_{j}(x) .
\end{aligned}
$$

Assumption 1 implies that, for faults $f \in \mathcal{F}_{i}$, the controller $u_{i}(t)$ stabilizes the plant as in (3). For faults $f \notin \mathcal{F}_{i}, V_{i}$ may increase, which implies that $x$ may escape to a large region or infinity as in (4). Section 4 will consider the case that no individual controller satisfies the specification (3) for the faulty plant.

The inequalities (2)-(5) distinguish the system's behavior under different controllers, which will play the key role in supervisory FDI/FTC. Other forms of $V_{i}$ than (2)(4) can also be defined, e.g., the dissipative form (Jiang et al., 2010) or the $\mathcal{K}_{\infty}$ function form (Yang et al., 2009).

3.1. Fault detection. The initial system is always regarded as a fault-free system, i.e., $f \in \mathcal{F}_{N}=\{0\}$, the applied controller being always $u_{N}$ at $t=0$. The system under the controller $u_{N}$ satisfies (3). In the presence of full state measurements, the inequality (3) can be naturally used as a time-varying residual to detect the fault as follows:

$$
V_{N}(x(t))>e^{-\lambda_{0} t} V_{N}(x(0)) \Longrightarrow \text { Fault occurs. }
$$

Fault detection can be achieved by the controller itself without requiring any individual fault detection scheme. The faults that do not violate (3) with $i=N$ are not necessary to be detected since they do not destroy stability. Denote $t_{f d}$ as the first time at which the inequality (3) is violated. Note that $x\left(t_{f d}\right)$ is still bounded.

Such a fault detection scheme is available even when the fault occurs from the beginning. In that case, (3) may be violated at the beginning and the fault is detected.

3.2. Fault isolation and supervisory FTC. For a switched system, to avoid arbitrary fast switchings, a "dwell-time" $\tau>0$ is often required such that the period between any two switching instants is no less than $\tau$. This implies that there is a finite number of switchings on any finite time interval. Such a "dwell-time" is also involved among controller switchings.

A performance based controller switching law is designed as follows.

\section{Algorithm 1.}

1. Set $t_{0}=t_{f d}$. Let $s=0$. Define $\Omega^{\star} \triangleq \Omega-\left\{\sigma\left(t_{f}\right)\right\}$. Set $\sigma\left(t_{0}\right)=i^{\star}$, where

$$
i^{\star}=\arg \max _{i \in \Omega^{\star}} J_{i}\left(x\left(t_{0}\right), t_{0}\right) .
$$

2. Choose $t_{1+s}=t_{s}+\tau$. If

$$
\dot{V}_{\sigma\left(t_{s}\right)}\left(x\left(t_{1+s}\right)\right) \leq-\lambda_{0} V_{\sigma\left(t_{s}\right)}\left(x\left(t_{s}\right)\right)
$$

then apply the controller $u_{\sigma\left(t_{s}\right)}(t), \forall t \geq t_{1+s}$. Stop the switching.

Otherwise, go to Step 3.

3. Let $\Omega^{\star}=\Omega^{\star}-\left\{\sigma\left(t_{s}\right)\right\}$. Set $\sigma\left(t_{1+s}\right)=i^{\star}$, where

$$
i^{\star}=\arg \max _{i \in \Omega^{\star}} J_{i}\left(x\left(t_{1+s}\right), t_{1+s}\right) .
$$

Apply the controller $u_{\sigma\left(t_{1+s}\right)}(t)$ at $t=t_{1+s}$.

Let $s=s+1$. Go to Step 2 .

The main idea behind Algorithm 1 is illustrated in Fig. 3. At each switching instant, we select the next controller that optimizes the given cost function from the set $\Omega^{\star}$. If this is the correct controller, then we apply it and then stop the switching (Step 2). Otherwise, we remove this destabilizing controller from $\Omega^{\star}$ (Step 3). The inequalities (3) and (4) are essentially "filters" for the overall FDI/FTC design (see Step 2) rather than FDI only.

The transient behavior during the switching delay obviously depends on $J_{i}(x(t), t), i \in \mathcal{M}^{\star}$. A few examples of relevant costs are given:

1. $J_{i}(x(t), t)$ is the probability that fault $i$ occurs in state $x(t)$ at time $t$. The switching policy selects the most likely fault mode.

2. $J_{i}(x(t), t)$ is a control cost that we wish to minimize if fault mode $i$ occurs. If the state escapes far away from its nominal trajectory, the control cost to bring back to its reference trajectory may be very large. The switching policy assumes that the worst fault mode has occurred, and selects first the associated control. The sooner the worst situation is recognized, the smaller the risk of an excessive control cost. On the contrary, the "optimistic" switching policy $i^{\star}=\arg \min _{i \in \mathcal{M}} J_{i}(x(t), t)$ is based on the occurrence of the best fault mode.

3. $i^{\star}=\operatorname{Random}\left\{i \in \mathcal{M}^{\star}(t)\right\}$ is always a possible option if no cost function can be elaborated. 


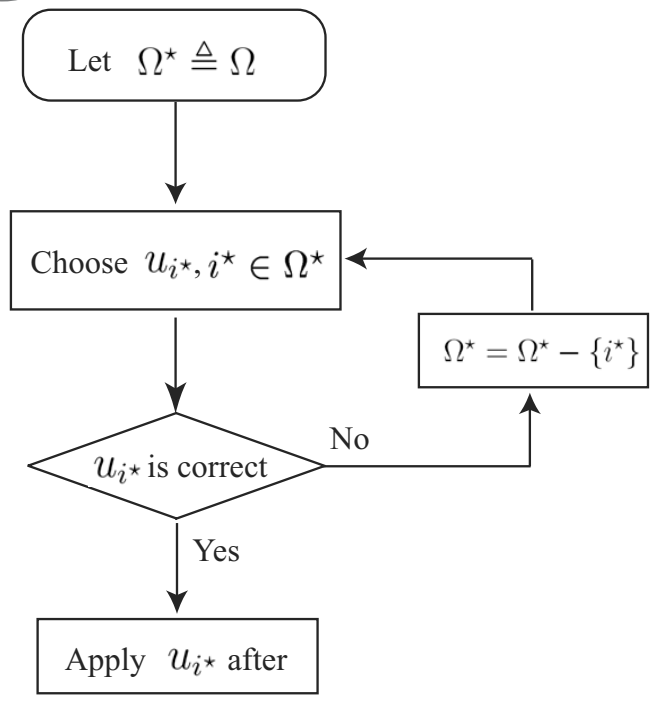

Fig. 3. Supervisory FTC algorithm.

Theorem 1. Consider the system (I) and a family of controllers satisfying Assumption 1. Suppose that a fault $f \in \mathcal{F}_{\iota}, \iota \in \mathcal{N}$ occurs at $t=t_{f}$. The fault detection law (6) and Algorithm 1 guarantee the stability of the origin.

Proof. Under Algorithm 1, at most $N-1$ switchings occur before the controller $u_{\iota}(t)$ related to $f \in \mathcal{F}_{\iota}$ is applied. We consider the worst case, i.e., $\sigma\left(t_{N-2}\right)=\iota$. The results for other cases are easily obtained. it follows

For two time instants $t$ and $t_{0}$, from (3) and (4) that

$$
\begin{aligned}
u=u_{i}, f \in \mathcal{F}_{i} & \Longrightarrow \\
V_{i}(x(t)) & \leq e^{-\lambda_{0}\left(t-t_{0}\right)} V_{i}\left(x\left(t_{0}\right)\right), \\
u=u_{i}, f \in \mathcal{F}_{j}, j & \neq i \Longrightarrow \\
V_{i}(x(t)) & \leq e^{\lambda_{1}\left(t-t_{0}\right)} V_{i}\left(x\left(t_{0}\right)\right) .
\end{aligned}
$$

Consider $t \in\left[t_{N-2}, \infty\right)$. Based on (5), (7) and (8), we further have

$$
\begin{aligned}
& V_{\iota}(x(t)) \\
& \leq \mu_{0} e^{-\lambda_{0}\left(t-t_{N-2}\right)} V_{\sigma\left(t_{N-2}^{-}\right)}\left(x\left(t_{N-2}\right)\right) \\
& \leq \mu_{0}^{2} e^{-\lambda_{0}\left(t-t_{N-2}\right)} e^{\lambda_{1} \tau} V_{\sigma\left(t_{N-3}^{-}\right)}\left(x\left(t_{N-3}\right)\right) \\
& \quad \vdots \\
& \leq \mu_{0}^{N-1} e^{-\lambda_{0}\left(t-t_{N-2}\right)} e^{(N-2) \lambda_{1} \tau} V_{\sigma\left(t_{0}^{-}\right)}\left(x\left(t_{0}\right)\right) .
\end{aligned}
$$

Since $N$ and $\tau$ are bounded, so is $x\left(t_{0}\right)=x\left(t_{f d}\right)$. From (96) it follows that $|x(t)|, t \geq t_{f d}$ is always bounded and $\lim _{t \rightarrow \infty} x(t)=0$. This completes the proof.

The switching process resulting from Algorithm 1 is equivalent to a switched system where some unstable modes are activated one by one and finally a stable mode
Table 1. System situations.

\begin{tabular}{|l||l|}
\hline Case 1 & $k_{1} \in[1,1.5), k_{2}=k_{3}=1$ \\
\hline Case 2 & $k_{2} \in(1,1.5], k_{1}=k_{3}=1$ \\
\hline Case 3 & $k_{3} \in[0.8,1), k_{1}=k_{2}=1$ \\
\hline Case 4 & $k_{1}=k_{2}=k_{3}=1$ \\
\hline
\end{tabular}

is activated. Therefore, the proof of Theorem 1 essentially relies on the stability analysis of the equivalent switched system.

As for a switched system with unstable modes, the overall stability can be guaranteed if the activating period of stable modes is long enough compared with that of unstable modes (Yang et al., 2009). Correspondingly, the stability under Algorithm 1 can be guaranteed if the activating period of destabilizing controllers is short enough (each destabilizing controller is activated for a minimal period $\tau$ ), and the stabilizing controller is activated long enough (being always activated after it is selected) as shown in Fig. 4. The switching algorithms related to the dissipative form and $\mathcal{K}_{\infty}$ function forms of $V_{i}$ can be found respectively in the works of Jiang et al. (2010) and Yang et al. (2009).

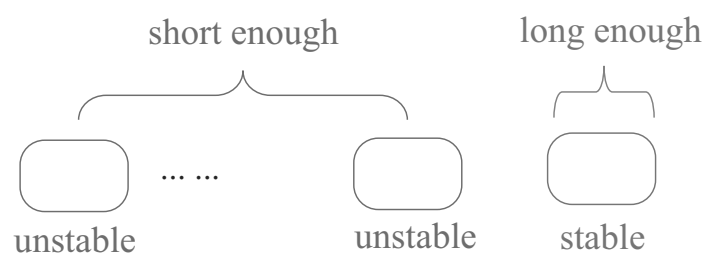

Fig. 4. Activating periods of different controllers.

3.3. Aircraft example. Fast and accurate flight control reconfiguration is of paramount importance for increasing aircraft survivability. The aircraft longitudinal differential equations under a small attack angle are expressed as $(\mathrm{Mu}$ et al., 2008)

$$
\left\{\begin{array}{l}
\dot{\vartheta}=\omega, \\
\dot{\omega}=k_{1} \eta^{\omega} \omega+k_{2} \eta^{\vartheta} \cos (\vartheta-\alpha)+k_{3} \eta u,
\end{array}\right.
$$

where the states $x=\left[\begin{array}{ll}\vartheta & \omega\end{array}\right]^{\top}$ denote the pitch angle and the pitch rate, respectively. Here $\alpha$ denotes the small attack angle. The input $u$ is the elevator deflection angle. $\eta^{\omega}, \eta^{\vartheta}$, and $\eta$ are longitudinal dynamics parameters chosen as $\eta^{\omega}=20(1 / s), \eta^{\vartheta}=-5\left(1 / s^{2}\right), \eta=-50\left(1 / s^{2}\right)$. Finally, $k_{1}, k_{2}$ and $k_{3}$ are fault coefficients. In the healthy situation, $k_{1}=k_{2}=k_{3}=1$.

Tables 1 and 2 describe system situations and their corresponding controllers.

Cases 1 and 2 deal with process faults, Case 3 is related to the actuator one, and Case 4 is a healthy sit- 
Table 2. Candidate controllers.

\begin{tabular}{|l|}
\hline$u_{1}=\frac{1}{\eta}\left[-1.5 \eta^{\omega} \omega-\eta^{\vartheta} \cos (\vartheta-\alpha)-5 \omega-5 \vartheta\right]$ \\
\hline$u_{2}=\frac{1}{\eta}\left[-\eta^{\omega} \omega-\operatorname{sgn}(\omega) 1.5 \eta^{\vartheta} \cos (\vartheta-\alpha)-5 \omega-5 \vartheta\right]$ \\
\hline$u_{3}=\frac{1.25}{\eta}\left[-\eta^{\omega} \omega-\operatorname{sgn}(\omega) \eta^{\vartheta} \cos (\vartheta-\alpha)-5 \omega-5 \vartheta\right]$ \\
\hline$u_{4}=\frac{1}{\eta}\left[-\eta^{\omega} \omega-\eta^{\vartheta} \cos (\vartheta-\alpha)-5 \omega-5 \vartheta\right]$ \\
\hline
\end{tabular}

uation. Consequently, we divide $\mathcal{F}$ into four parts as $\mathcal{F} \subset \bigcup_{i \in \mathcal{M}=\{1,2, \ldots, 4\}} \mathcal{F}_{i}$, where $\mathcal{F}_{i}$ is related to the fault values in Case $i . \mathcal{F}_{4}$ denotes the fault-free situation.

In the simulation, suppose that Case 1 occurs at $t=$ 1.5 s. Set $k_{1}=1.5$. Define $V(x)=x^{\top} P x$, with

$$
P=\left[\begin{array}{cc}
11 & 1 \\
1 & 1.2
\end{array}\right]
$$

We further have

$$
\begin{aligned}
& \dot{V}(x) \leq-10 V(x), \quad \forall f \in \mathcal{F}_{1}, u=u_{1} \\
& \dot{V}(x) \leq 17.6205 V(x), \quad \forall f \in \mathcal{F}_{1}, u=\left\{u_{2}, u_{3}, u_{4}\right\}
\end{aligned}
$$

which satisfy Assumption 1. The system under the faulty Case 1 is stabilized only by the controller $u_{1}(x)$. Suppose that the initial states are $[0.1(\mathrm{rad}) \quad 0.2(\mathrm{rad} / \mathrm{s})]^{\top}$. Figure 5 shows that the fault is detected rapidly at $t=1.5 \mathrm{~s}$ using the threshold (6).

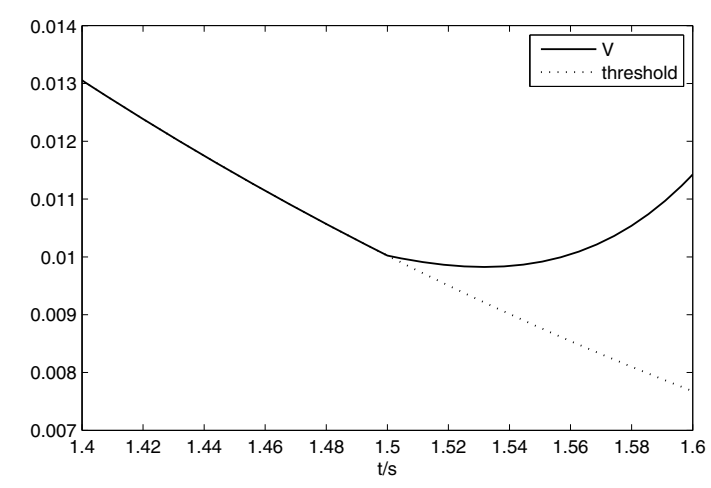

Fig. 5. Fault detection.

Now we apply Algorithm 1 to achieve the FTC objective. Given the cost function $J_{i}(x(t))=\int_{0}^{t} x^{2}(s)+$ $0.1 u_{i}^{2}(s) \mathrm{d} s$, the optimal switching sequence obtained is $u_{2} \rightarrow u_{3} \rightarrow u_{1}$. Choose $\tau=0.5 \mathrm{~s}$. Then $u_{2}(x)$ is applied at $t=1.5 \mathrm{~s}$, and switches to $u_{3}(x)$ at $t=2 \mathrm{~s}$. Then $u_{1}(x)$ is selected and applied at $t=2.5 \mathrm{~s}$. The fault is identified to be Case 1 . The correct controller $u_{1}(x)$ is then applied for $t \geq 2.5 \mathrm{~s}$. Figure 6 shows the state and input trajectories. It can be seen that the FTC goal is achieved, the states are always bounded, and the control magnitude is not large.

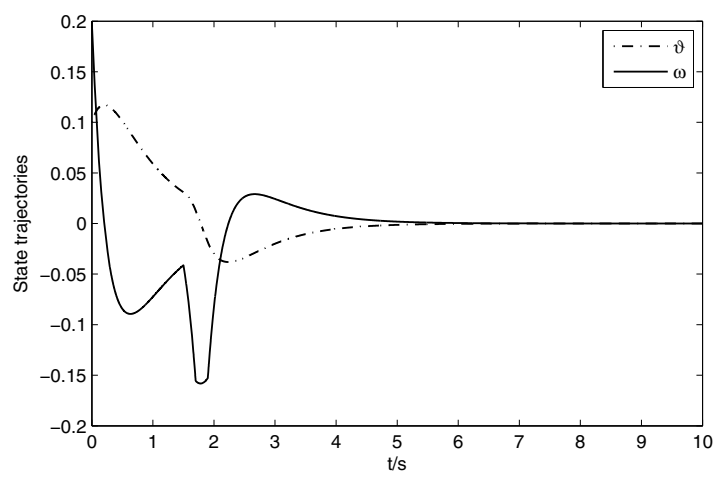

Fig. 6. State and input trajectories.

\section{Supervisory FTC: Relaxation of L1-L3}

The supervisory FTC scheme developed in Section 3 relaxes L1-L2, while L3 is still assumed, i.e., each possible fault set can be accommodated by at least one of candidate controllers. In this section, we further relax L3 and consider the case when some faults cannot be accommodated by any individual candidate controller, but can be accommodated by a set of controllers under an appropriate switching among them.

The main supervisory FTC idea is similar to that in Section 3. However, since there is no individual controller to accommodate the fault, the switching periodically works among a set of candidate controllers related to the current fault and never stops. The controller switching process is equivalent to a switched system with all unstable modes.

For clarity, we first discuss the switched system in Section 4.1, and then apply the result to supervisory FTC in Section 4.2.

4.1. Stabilization of switched systems with all unstable modes. Let us consider a switched system where all modes may be unstable. The main idea is to divide states into several parts. Consequently, the original switched system is regarded as a set of interconnected sub-switched systems. Under some conditions, each sub-switched system is input-to-state stable with respect to states of the others. This, together with small gain conditions, leads to the asymptotical stability of the overall system.

The switched system takes the form

$$
\dot{x}=f_{\sigma}\left(x, u_{\sigma}\right),
$$

where $x \in \mathbb{R}^{n}$ are the states. Define $\mathcal{M}=\{1,2, \ldots, m\}$, where $m$ is the number of modes. $\sigma(t):[0, \infty) \rightarrow \mathcal{M}$ denotes the switching signal, which is assumed to be a piecewise constant function continuous from the right. For any $i \in \mathcal{N}, u_{i} \in \mathbb{R}^{p}$ are the inputs and $f_{i}$ is a smooth function with $f_{i}(0,0)=0$. Denote by $\Delta t_{i}$ the activating period of mode $i$. The "dwell-time" $\tau$ is still involved. 
It is desirable to design $u_{\sigma}$ such that each mode of (10) is individually stable, which, however, is often hard for complex nonlinear structures. A natural question is whether the switched system can be stabilized by the appropriate design of $\sigma$ and $u_{\sigma}$. The answer is positive, as shown below.

Define a vector $z=\left[z_{1}^{\top}, z_{2}^{\top}, \ldots, z_{m}^{\top}\right]^{\top}, m \leq n$, satisfying

$$
\begin{aligned}
z_{1} & \in \mathbb{R}^{n_{1}}=\left[x_{1}, \ldots, x_{n_{1}}\right]^{\top} \\
z_{2} \in \mathbb{R}^{n_{2}} & =\left[x_{n_{1}+1}, \ldots, x_{n_{1}+n_{2}}\right]^{\top} \\
\vdots & \\
z_{m} \in \mathbb{R}^{n_{m}} & =\left[x_{\sum_{i=1}^{m-1}\left(n_{i}\right)+1}, \ldots, x_{\sum_{i=1}^{m}\left(n_{i}\right)}\right]^{\top},
\end{aligned}
$$

where $\sum_{i=1}^{m}\left(n_{i}\right)=n$. It is clear that the original states $x$ are divided into $m$ parts by $z$.

Assumption 2. For each mode $i \in \mathcal{M}$ of the system (10), we can design a controller $u_{i}$ under which there exists a continuous non-negative function

$$
V^{\langle i\rangle}: \mathbb{R}^{n} \rightarrow \mathbb{R}_{\geq 0}=V_{1}^{\langle i\rangle}\left(z_{1}\right)+\cdots+V_{m}^{\langle i\rangle}\left(z_{m}\right),
$$

where $V_{k}^{\langle i\rangle}\left(z_{k}\right) \in \mathcal{C}^{1}: \mathbb{R}^{n_{k}} \rightarrow \mathbb{R}_{\geq 0}, k \in \mathcal{M}$, and there exist $\alpha_{1}, \alpha_{2} \in \mathcal{K}_{\infty}$, and $\gamma_{a b} \in \mathcal{K}_{\infty}$, for $a, b \in \mathcal{M}$, $\lambda_{0}, \lambda_{1}>0, \mu \geq 1$ such that $\forall i, p, q, l \in \mathcal{M}$,

$$
\begin{aligned}
& \alpha_{1}\left(\left|z_{k}\right|\right) \leq V_{k}^{\langle i\rangle}\left(z_{k}\right) \leq \alpha_{2}\left(\left|z_{k}\right|\right), \quad \forall k \in \mathcal{M}, \\
& \dot{V}_{i}^{\langle i\rangle}\left(z_{i}\right) \leq-\lambda_{0} V_{i}^{\langle i\rangle}\left(z_{i}\right)+\max _{p \in \mathcal{M}-\{i\}}\left\{\gamma_{i p}\left(V_{p}^{\langle i\rangle}\right)\right\} \\
& \dot{V}_{j}^{\langle i\rangle}\left(z_{j}\right) \leq \lambda_{1} V_{j}^{\langle i\rangle}\left(z_{j}\right)+\max _{q \in \mathcal{M}-\{j\}}\left\{\gamma_{j q}\left(V_{q}^{\langle i\rangle}\right)\right\} \\
& \forall j \in \mathcal{M}-i, \\
& V_{l}^{\langle p\rangle}\left(z_{l}\right) \leq \mu V_{l}^{\langle q\rangle}\left(z_{l}\right) .
\end{aligned}
$$

The inequalities (14) and (15) imply that, for mode $i$, $z_{i}$ is Input-to-State Stable (ISS) (Sontag and Wang, 1996) with respect to other states of $z, \gamma_{a b}$ is the gain from $V_{b}^{\langle i\rangle}$ to $V_{a}^{\langle i\rangle}$, while all other states of $z$ may not be stable. Here $z_{i}$ is called a potentially stable state in mode $i$.

Although each mode $i$ cannot be stabilized, Assumption 2 guarantees that each mode has some potentially stable states under appropriate $u_{i}$, and all these potentially stable states in different modes form the whole state space.

Under Assumption 2, the switched system (10) can be regarded as $m$ interconnected switched systems as shown in Fig. 7. We call each switched system a $z_{i}$ switched system.

Definition 1. A periodical switching signal for the system 10] is given by

$$
\begin{array}{r}
\sigma(t)=l, \quad \forall t \in\left[k T+\sum_{\rho=0}^{l-1} \Delta t_{\rho}, k T+\sum_{\rho=0}^{l} \Delta t_{\rho}\right), \\
k=0,1, \ldots,
\end{array}
$$

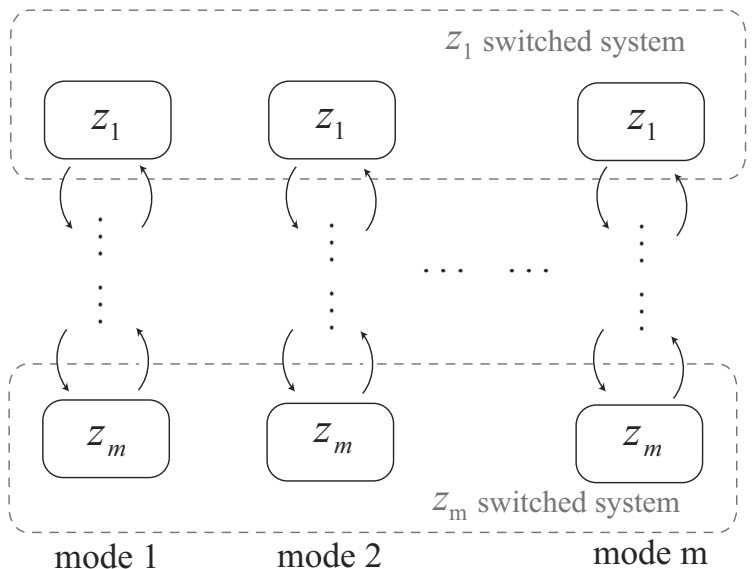

Fig. 7. Structure of switched systems.

where $T=\sum_{l=1}^{m} \Delta t_{l}$ is one period of the periodical switching sequence, and $\Delta t_{l} \geq \tau$ is the activating period of mode $l$ in each period.

Definition 2. The switched system (10) is periodically stabilizable if there exists a periodical switching signal $\sigma$ under which the origin of the switched system (10) is asymptotically stable, i.e., $\lim _{t \rightarrow \infty} x(t)=0$.

Lemma 1. For any $z_{i}$ switched system, $i \in \mathcal{M}$, satisfying Assumption 2, if there exists a periodical switching signal such that $\Delta t_{u s}, \Delta t_{i} \geq \tau$ and

$$
(m-1) \ln \mu+\Delta t_{u s} \lambda_{1}<\Delta t_{i} \lambda_{0}
$$

with $\Delta t_{i} \geq \tau, \Delta t_{u s}=\left(\sum_{l=1}^{m} \Delta t_{l}\right)-\Delta t_{i}$, then a $z_{i}$ switched system is ISS with respect to other states of $z$, i.e.,

$$
\left|z_{i}(T)\right| \leq \beta\left(\left|z_{i}(0)\right|, T\right)+\max _{k \in \mathcal{M}-\{i\}}\left\{\bar{\gamma}_{i k}\left(\left\|z_{k}\right\|_{[0, T)}\right)\right\},
$$

where $\beta \in \mathcal{K} \mathcal{L}, \bar{\gamma}_{i b} \in \mathcal{K}_{\infty}$ for $b \in \mathcal{M}$.

Proof. The proof can be obtained following the same line as (Yang et al., 2009).

The condition 177 is illustrated in Fig. 8, which means that for each $z_{i}$ switched system, if the activating period of mode $i$ is large enough compared with that of other modes in one period $T$, the overall $z_{i}$ switched system is ISS with respect to other states of $z$ at $T$. For

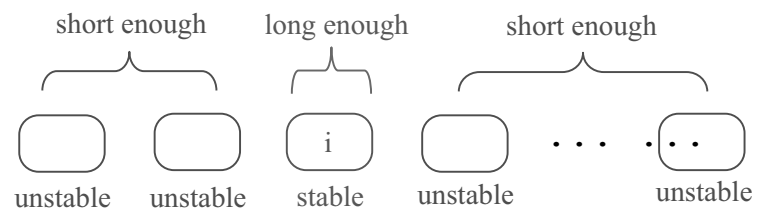

Fig. 8. Activating periods of different modes. 
$a, b \geq \tau$ define

$$
\left\{\begin{array}{l}
\phi_{1}(a) \triangleq \frac{\lambda_{0} a-(m-1) \ln \mu}{\lambda_{1}} \\
\phi_{2}(b) \triangleq \frac{\lambda_{1} b+(m-1) \ln \mu}{\lambda_{0}}
\end{array}\right.
$$

The condition (17) is equivalent to $\Delta t_{u s}<\phi_{1}\left(\Delta t_{i}\right)$ and $\Delta t_{i}>\phi_{2}\left(\Delta t_{u s}\right)$. The following theorem gives the sufficient stability conditions of the overall switched system.

Theorem 2. Under Assumption 2, if

1. there exists $\Delta t \geq \tau$, such that

$$
\phi_{2}\left(\Delta t+\phi_{1}(\Delta t)\right) \geq \tau
$$

and

$$
\phi_{1}(\Delta t)>(m-1) \phi_{2}\left(\Delta t+\phi_{1}(\Delta t)\right)
$$

2. there exists $\rho \in \mathcal{K}_{\infty}$, such that

$$
\left(\bar{\gamma}_{i_{1} i_{2}}+\rho\right) \circ\left(\bar{\gamma}_{i_{2} i_{3}}+\rho\right) \circ \cdots \circ\left(\bar{\gamma}_{i_{r} i_{1}}+\rho\right)(s) \leq s
$$

for all $s \geq 0$, and for all $1 \leq i_{j} \leq m, i_{j} \neq i_{j^{\prime}}$ whenever $j \neq j^{\prime}$,

then the switched system (10) is periodically stabilized by the following switching law:

$$
T=\Delta t+(m-1) \phi_{2}\left(\Delta t+\phi_{1}(\Delta t)\right) .
$$

\section{Algorithm 2.}

1. Let $k=0$.

2. Activate Mode 1 at $t=k T$, until $t=k T+\Delta t$. Set $i=2$, and go to Step 3 .

3. Activate Mode $i$ at

$$
t=k T+\Delta t+(i-2) \phi_{2}\left(\Delta t+\phi_{1}(\Delta t)\right),
$$

until

$$
t=k T+\Delta t+(i-1) \phi_{2}\left(\Delta t+\phi_{1}(\Delta t)\right) .
$$

Go to Step 4.

4. Set $i=i+1$. If $i=m+1$, then $k=k+1$.

Go to Step 2. Otherwise, go to Step 3.

Proof. It can be easily obtained from Lemma 1 that, under the first condition, Algorithm 2 guarantees that all $z_{i}$ switched systems are ISS with respect to other states of $z$, i.e., $\forall i \in \mathcal{M}$, the inequality (18) holds at $T$. On the other hand, the second condition means that the composition of the gain function along every closed cycle among interconnected $z_{i}$ systems is less than the identity function. Also note that all potentially stable states in different modes form the whole state space. It follows from the works of Jiang et al. (1994) as well as Jiang and Wang (2008) that the origin of the switched system (10) is asymptotically stable at $T$, i.e., there exists $\beta^{\star} \in \mathcal{K} \mathcal{L}$ such that $|x(T)| \leq \beta^{\star}(|x(0)|, T)$. Proceeding in a similar way, we obtain

$$
|x((k+1) T)| \leq \beta^{\star}(|x(k T)|, T), \quad k=0,1,2, \ldots .
$$

Therefore, the origin of the switched system (10) is asymptotically stable under Algorithm 2.

The main idea of Theorem 2 is to guarantee that each $z_{i}$ switched system has enough time to activate modes that have potentially stable states, as illustrated in Fig. 9, which, together with the second condition of Theorem 2 (the small gain condition), leads to the asymptotical stability of the overall switched system at the origin.

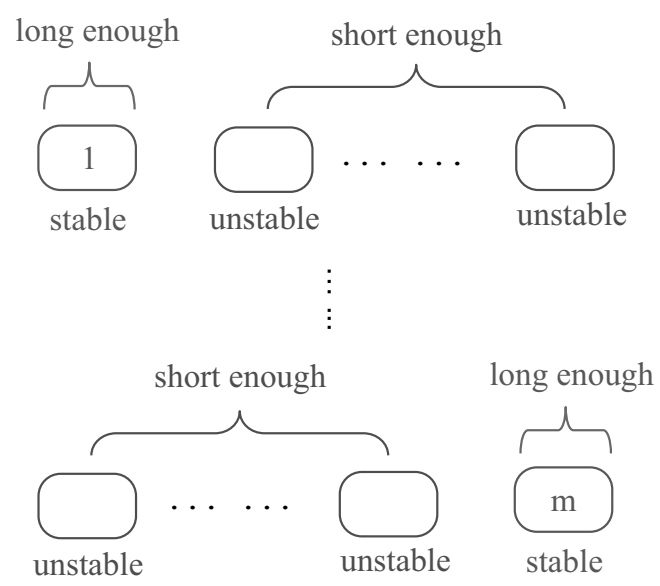

Fig. 9. Activating periods of different modes.

4.2. Supervisory FTC. This section applies the above results to a supervisory FTC design problem. Recall the system (1). Divide $x$ into $m$ parts by $z$ as in (11). It is still supposed that there are $\omega$ pre-computed candidate controllers for the supervision purpose. Recall that $\Omega=\{1,2, \ldots, \omega\}$.

Assumption 3. There exist $m$ candidate controllers ( $m \leq \omega)$, denoted by $u_{i}, i \in \mathcal{M}$, such that when the system (17) experiences the fault $f \in \mathcal{F}_{\iota}, \iota \in \mathcal{N}$, and $u=u_{i}$, there exists a continuous non-negative function $V^{\langle i\rangle}$ that satisfies (12)-(16). Moreover, if $u=u_{s}, s \in \Omega \backslash \mathcal{M}$, then $\forall j \in \mathcal{M}$,

$$
\dot{V}_{j}^{\langle i\rangle}\left(z_{j}\right) \leq \lambda_{1} V_{j}^{\langle i\rangle}\left(z_{j}\right)+\max _{q \in \mathcal{M}-\{j\}}\left\{\gamma_{j q}\left(V_{q}^{\langle i\rangle}\right)\right\},
$$

where $\lambda_{1}$, and $\gamma_{a b}$ are defined as in Assumption 2. 
Assumption 3 implies that, for $f \in \mathcal{F}_{\iota}$, each controller $u_{i}, i \in \mathcal{M}$, may potentially stabilize some states, and all potentially stable states under these $m$ controllers compose the whole state space. However, when any other candidate controller $u_{i}, i \in \Omega \backslash \mathcal{M}$, is applied, the system has no potentially stable state.

It should be pointed out that Assumption 3 covers the case when the healthy system (if $\iota=M$ ) can neither be stabilized by any individual candidate controller. This is often true in some kinds of systems, e.g., underactuated ones, while the switching control scheme can achieve the stability objective.

The fault detection law can be designed in much the same way as in Section 3. Once $u_{i}$ is applied, (14) can be used as a time-varying residual, and a fault detection law is given by

$$
\begin{aligned}
V_{i}^{\langle i\rangle}(t)> & e^{-\lambda_{0}\left(t-t_{i k}\right)} V_{i}^{\langle i\rangle}\left(t_{i k}\right) \\
& +\int_{t_{i k}}^{t} e^{-\lambda_{0}(t-\nu)} \max _{p \in \mathcal{M}-\{i\}}\left\{\gamma_{i p}\left(V_{p}^{\langle i\rangle}(\nu)\right)\right\} \mathrm{d} \nu \\
& \Longrightarrow \text { Fault occurs }
\end{aligned}
$$

where $t_{i k}$ denotes the $k$-th time at which the controller $u_{i}(t)$ is applied. Suppose that the fault is detected at $t=$ $t_{f d}$. For simplification, we only focus on one fault set $\mathcal{F}_{\iota}$, for $\iota \in \mathcal{N}$. The results can be easily extended to the of multiple faults considered.

In the following, a $z_{i}$ switched system is considered with the dynamics of $z_{i}$ under different controllers. To avoid arbitrarily fast switchings, the "dwell-time" $\tau$ is also involved among controller switchings.

Define $T=\Delta t+(m-1) \phi_{2}\left(\Delta t+\phi_{1}(\Delta t)\right)$ with $\Delta t \geq \tau$ to be designed, $\phi_{1}$ and $\phi_{2}$ being defined in (19). A performance based controller switching law is designed as follows.

\section{Algorithm 3.}

1. Define $\Omega^{\star} \triangleq \Omega$. Let $s=0, k=0, v=1$.

Set $\sigma\left(t_{f d}\right)=i^{\star}$, where

$$
i^{\star}=\arg \min _{i \in \Omega^{\star}} J_{i}\left(x\left(t_{f d}\right), t_{f d}\right) .
$$

2. Apply $u_{i^{\star}}$ until $t=t_{f d}+(s+1) \tau$.

If there is a $j \in \mathcal{M}$ such that $V_{j}^{<i^{\star}>}$ satisfies (14), then go to Step 4. Otherwise, go to 3.

3. Let $\Omega^{\star}=\Omega^{\star} \backslash\left\{i^{\star}\right\}$. Set $\sigma\left(t_{f d}+(s+1) \tau\right)=i^{\star}$ where

$$
i^{\star}=\arg \min _{i \in \Omega^{\star}} J_{i}\left(x\left(t_{f d}+(s+1) \tau\right), t_{f d}+(s+1) \tau\right) .
$$

Let $s=s+1$. Go to Step 2 .

4. Let $\Omega^{\star}=\mathcal{M}$. Apply $u_{i^{\star}}$ until

$$
t=t_{f d}+k T+s \tau+\Delta t .
$$

5. Let $\Omega^{\star}=\Omega^{\star} \backslash\left\{i^{\star}\right\}$. Set $\sigma(t)=i^{\star}$, where

$$
i^{\star}=\arg \min _{i \in \Omega^{\star}} J_{i}(x(t), t) .
$$

Apply $u_{i^{\star}}$ until

$$
t=t_{f d}+k T+s \tau+\Delta t+v \phi_{2}\left(\Delta t+\phi_{1}(\Delta t)\right) .
$$

Let $v=v+1$. If $v=m$, let $k=k+1$, go to Step 4 . Otherwise, go to Step 5.

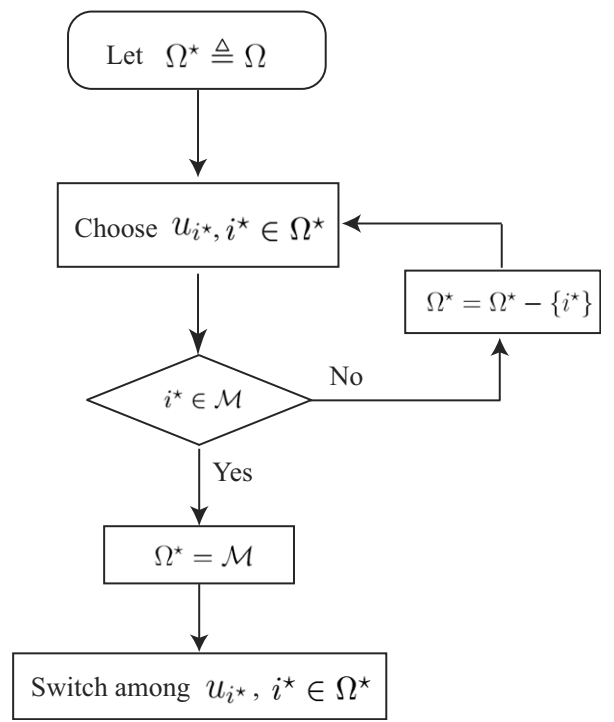

Fig. 10. Supervisory FTC algorithm.

The main idea of Algorithm 3 is shown in Fig. 10 We first choose one controller from among all candidate ones whose related cost function $J_{i}$ is minimal (Step 1). If the current controller is $u_{i}, i \in \Omega \backslash \mathcal{M}$ (Step 2), then exclude this controller from candidate ones and continue choosing another controller. If the current controller is $u_{i}$, $i \in \mathcal{M}$, under which the system with $f \in \mathcal{F}_{\iota}$ has potential stable states, and, meanwhile, the current faulty situation is identified (Step 2), then a performance based periodical switching will occur among the corresponding $u_{i}, i \in \mathcal{M}$ (Steps 4 and 5), and any other controller $u_{i}, i \in \Omega \backslash \mathcal{M}$, will never be applied.

Theorem 3. Consider the system (1) with $f \in \mathcal{F}_{\iota}$ and a family of controllers satisfying Assumption 3. The fault detection law (22) and Algorithm 3 make the origin of the system asymptotically stable if there exists $\Delta t \geq \tau$ such that $\phi_{2}\left(\Delta t+\phi_{1}(\Delta t)\right) \geq \tau$, and

$$
\phi_{1}(\Delta t)>(m-1) \phi_{2}\left(\Delta t+\phi_{1}(\Delta t)\right)+(\omega-m) \tau
$$

as well as the second condition of Theorem 2 holds.

Proof. Consider the worst case, i.e., when all controllers $u_{i}, i \in \Omega \backslash \mathcal{M}$, are applied one by one with activating 
period $\tau$. At $t=t_{f d}+(\omega-m) \tau$, one of the controllers $u_{i}, i \in \mathcal{M}$, denoted by $u_{1}$, is selected and applied. It follows from Algorithm 3 that the activating period of $u_{1}$ is $\Delta t$. All other consequent $m-1$ controllers have the activating period $(m-1) \phi_{2}\left(\Delta t+\phi_{1}(\Delta t)\right)$.

At $t=T^{\star} \triangleq t_{f d}+\Delta t+(m-1) \phi_{2}\left(\Delta t+\phi_{1}(\Delta t)\right)+$ $(\omega-m) \tau$, all controllers have been applied for one time. Based on Assumption 3 and (23), we can obtain, following the same reasoning as in the proof of Theorem 2, that all $z_{i}$ switched systems are ISS with respect to other states of $z$ at $T^{\star}$, which, together with the second condition in Theorem 1, leads to the asymptotical stability of the origin at $T^{\star}$. Note that (23) implies 20), the rest of the proof being the same as that of Theorem 2. For the case when $u_{1}$ is selected at $t=t_{f d}+(\bar{\omega}-m) \tau$, with $\bar{\omega}<\omega$, the result can be obtained following the above procedure.

4.3. Aircraft team example. In a team of multiple aircrafts, one leading aircraft often determines the flying behavior of the whole team. The others have no behavior information by themselves. The flying performance of the whole team is achieved by communications among aircrafts (Giulietti et al., 2000).

Specifically, in the "climbing" process, it is required that all aircrafts in the team have the same pitch rates. We consider a team consisting of three aircrafts as shown in Fig. 11. Aircraft 1 is the unique leader that knows the prescribed pitch rate. Two other aircrafts follow Aircraft 1 by receiving the state information from Aircraft 1.

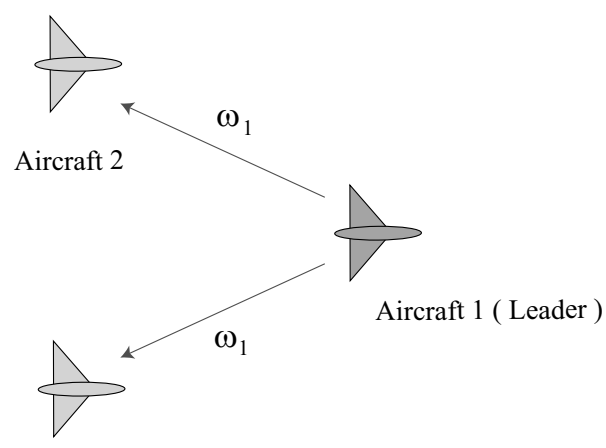

Aircraft 3

Fig. 11. Aircraft team.

The longitudinal differential equation of each aircraft is the same as in the example in Section 3.3. For the purpose of cooperation, we divide the original controller $u$ into the self controller $u^{s}$ and the cooperative controller $u^{c}$, i.e., $u=u^{s}+u^{c}$. Suppose that the dynamic equations of pitch rates under their self controllers are as follows:

$$
\begin{array}{ll}
\text { Aircraft 1: } & \dot{\omega}_{1}=-a_{1}\left(\omega_{1}-\omega^{\star}\right), \\
\text { Aircraft 2: } & \dot{\omega}_{2}=a_{2}\left(\omega_{2}-\omega^{\star}\right)+\underbrace{b_{2}\left(\omega_{1}-\omega_{2}\right)}_{u_{2}^{c}}, \\
\text { Aircraft 3: } & \dot{\omega}_{3}=a_{3}\left(\omega_{3}-\omega^{\star}\right)+\underbrace{b_{3}\left(\omega_{1}-\omega_{3}\right)}_{u_{3}^{c}},
\end{array}
$$

where $a_{1}, a_{2}, a_{3}, b_{1}, b_{2}$ are positive constants, and $2 a_{2}-$ $b_{2}<0,2 a_{3}-b_{3}<0$. It can be seen that Aircraft 1 does not need cooperation with the others, i.e., $u_{1}^{c}=0$, since it can approach the prescribed pitch rate $\omega^{\star}$ by itself. However, without information from Aircraft 1, pitch rates of Aircrafts 2 and 3 may run far away from $\omega^{\star}$. Here $u_{2}^{c}$ and $u_{3}^{c}$ just play the cooperation role.

Define $W_{i}=\left(\omega_{i}-\omega^{\star}\right)^{2}, i=1,2,3$. Differentiating $W_{i}$ along (24)-(26), we further have

$$
\left\{\begin{array}{l}
\dot{W}_{1}=-2 a_{1} W_{1}, \\
\dot{W}_{2} \leq\left(2 a_{2}-b_{2}\right) W_{2}+b_{2} W_{1} \\
\dot{W}_{3} \leq\left(2 a_{3}-b_{3}\right) W_{3}+b_{3} W_{1} .
\end{array}\right.
$$

This implies that all $\omega_{i}$ will approach $\omega^{\star}$.

Poor link quality is an inherit drawback of wireless communication, which often leads to great transmission power and a large number of retransmissions of sensors, and consequently, a drastically increasing communication cost (Akyildiz et al., 2002). Now we consider a communication fault case, i.e., that transmitter of Aircraft 1 is faulty, such that it does not have enough power to transmit information in a region as large as the healthy one. Thus, Aircrafts 2 and 3 cannot receive the information from Aircraft 1 simultaneously. Communication between Aircrafts 2 and 3 is also supposed to be unavailable. In such a faulty case, Aircraft 1 can send information to one aircraft only. Any fixed connection topology cannot achieve the team flight. However, a switching topology can do it.

Under Topology 1: Connecting Aircraft 2 with Aircraft 1 yields

$$
\left\{\begin{array}{l}
\dot{W}_{1}=-2 a_{1} W_{1} \\
\dot{W}_{2} \leq\left(2 a_{2}-b_{2}\right) W_{2}+b_{2} W_{1} \\
\dot{W}_{3}=2 a_{3} W_{3} .
\end{array}\right.
$$

In this situation, $\omega_{1}$ and $\omega_{2}$ will tend to $\omega^{\star}$, but $\omega_{3}$ may run far away.

Under Topology 2: Connecting Aircraft 3 with Aircraft 1 yields

$$
\left\{\begin{array}{l}
\dot{W}_{1}=-2 a_{1} W_{1} \\
\dot{W}_{2}=2 a_{2} W_{2} \\
\dot{W}_{3} \leq\left(2 a_{3}-b_{3}\right) W_{3}+b_{3} W_{1} .
\end{array}\right.
$$

In this situation, $\omega_{1}$ and $\omega_{3}$ reaches $\omega^{\star}$, while $\omega_{2}$ may escape. 
Note that (28) and (29) inherently satisfy (13)-16) in Assumption 2. In the simulation, suppose that $a_{1}=5$, $a_{2}=a_{3}=2, b_{2}=b_{3}=10$. We further have

$$
\begin{aligned}
& \text { Topology 1: }\left\{\begin{array}{l}
\dot{W}_{1}=-10 W_{1}, \\
\dot{W}_{2}=-6 W_{2}+10 W_{1}, \\
\dot{W}_{3}=4 W_{3} .
\end{array}\right. \\
& \text { Topology 2: }\left\{\begin{array}{l}
\dot{W}_{1}=-10 W_{1}, \\
\dot{W}_{2}=4 W_{2}, \\
\dot{W}_{3}=-6 W_{3}+10 W_{1} .
\end{array}\right.
\end{aligned}
$$

which satisfy 20) and 21) in Theorem 2.

Choose $\omega^{\star}=0.1(\mathrm{rad} / \mathrm{s})$. The initial states are $\omega_{1}(0)=0.3(\mathrm{rad} / \mathrm{s}), \omega_{2}(0)=0(\mathrm{rad} / \mathrm{s}), \omega_{3}(0)=-0.2$ $(\mathrm{rad} / \mathrm{s})$. Suppose that the fault occurs at $t=0.5 \mathrm{~s}$, at which both connections are broken. The fault can be detected rapidly at $t=0.5 \mathrm{~s}$ using 27.

Since there are only two topologies to be selected and applied, Algorithm 3 can be simplified and skips to Step 4. The cost functions are $J_{1}=\omega_{2}^{2}+\omega_{3}^{2}, J_{2}=\omega_{2}^{2}+\omega_{3}^{4}$. Topology 2 is firstly selected and applied at $t=0.5 \mathrm{~s}$ since $J_{2}(2)<J_{1}(2)$. Both dwell periods of Topologies 1 and 2 are chosen as $0.5 \mathrm{~s}$. The periodical switching is as follows:

$$
\begin{cases}\text { Topology 2: } & \forall t \in[0.5+k(s), 1+k(s)) \\ \text { Topology 1: } & \forall t \in[1+k(s), 1.5+k)(s))\end{cases}
$$

for $k=0,1,2, \ldots$.

Figure 12 shows the switching signal (where ' 1 ' represents Topology 1, and '2' denotes Topology 2) and pitch rate trajectories under the proposed supervisory FTC law, from which we can see that all pitch rates approach $\omega^{\star}$ in spite of the communication fault.
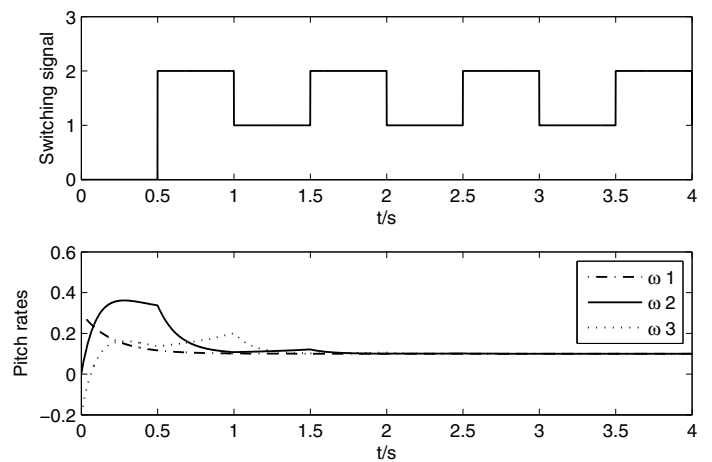

Fig. 12. Switching signal and pitch rate trajectories.

\section{Conclusion}

This paper provides a new supervisory FTC framework without individual fault detection and isolation schemes. The proposed framework only relies on a controller switching scheme which is based on switched system theories.

Future work will be conducted along the following lines:

1. In this work, full state measurements are available, which facilitates FDI/FTC design. In the absence of measurable states, an output feedback controller would be potentially applied, or observers would be embedded into the proposed framework. Switching law design would be challenging.

2. In Section 4, exponential decay form of $V_{i}$ is considered such that $\phi_{1}$ and $\phi_{2}$ are independent of the states. The state-dependent $\phi_{1}$ and $\phi_{2}$ would be considered. In this case, the stability of the system should be checked on-line, and switching law design is much more complicated.

3. The state may oscillate during the switching period of controllers due to a large switching number and switching frequency. The trade-off between the simplicity of the switching algorithm and its effects on the transient performance will be investigated.

\section{Acknowledgment}

This work is supported by the National Natural Science Foundation of China $(61104116,61034005)$ and the NUAA Research Funding (NZ2010003, NS2011016).

\section{References}

Akyildiz, I.F., Su, W., Sankarasubramaniam, Y. and Cayirci, E. (2002). Wireless sensor networks: A survey, Computer Networks 38(4): 393-422.

Blanke, M., Kinnaert, M., Lunze, J. and Staroswiecki, M. (2006). Diagnosis and Fault-Tolerant Control, 2nd Edn., Springer-Verlag, Berlin/Heidelberg.

Giulietti, F., Pollini, L. and Innocenti, M. (2000). Autonomous formation flight, IEEE Control Systems Magazine 20(6): 34-44.

Jiang, B., Yang, H. and Shi, P. (2010). Switching fault tolerant control design via global dissipativity, International Journal of Systems Science 41(8): 1003-1012.

Jiang, Z.P., Teel, A.R. and Praly, L. (1994). Small-gain theorem for ISS systems and applications, Mathematics of Control, Signals, and Systems 7(1): 95-120.

Jiang, Z.P. and Wang, Y. (2008). A generalization of the nolinear small-gain theorem for large-scale complex systems, Proceedings of the 7th World Congress on Intelligent Control and Automation, Chongqing, China, pp. 1188-1193.

$\mathrm{Mu}, \mathrm{X}$., Zhang, W. and Zhang, W. (2008). An adaptive backstepping design for longitudinal flight path control, Proceedings of the 7th World Congress on Intelligent Control and Automation, Chongqing, China, pp. 5249-5251. 
Parisini, T. and Sacone, S. (2001). Stable hybrid control based on discrete-event automata and receding-horizon neural regulators, Automatica 37(5): 1279-1292.

Patton, R.J., Frank, P.M. and Clark, R.N. (2000). Issues of Fault Diagnosis for Dynamic Systems, Spring-Verlag, London.

Sontag, E. and Wang, Y. (1996). New characterizations of inputto-state stability, IEEE Transactions on Automatic Control 41(9): 1283-1294.

Staroswiecki, M. and Gehin, A.-L. (2001). From control to supervision, Annual Reviews in Control 25(1): 1-11.

Yang, H., Cocquempot, V. and Jiang, B. (2009). On stabilization of switched nonlinear systems with unstable modes, Systems \& Control Letters 58(10): 703-708.

Yang, H., Jiang, B. and Cocquempot, V. (2009). A fault tolerant control framework for periodic switched nonlinear systems, International Journal of Control 82(1): 117-129.

Yang, H., Jiang, B. and Cocquempot, V. (2010). Fault Tolerant Control Design For Hybrid Systems, Springer-Verlag, Berlin/Heidelberg.

Yang, H., Jiang, B. and Staroswiecki, M. (2009). Supervisory fault tolerant control for a class of uncertain nonlinear systems, Automatica 45(10): 2319-2324.

Zhang, X., Polycarpou, M.M. and Parisini, T. (2008). Design and analysis of a fault isolation scheme for a class of uncertain nonlinear systems, Annual Reviews in Control 32(1): 107-121.

Zhang, Y.M. and Jiang, J. (2008). Bibliographical review on reconfigurable fault-tolerant control systems, Annual Reviews in Control 32(2): 229-252.

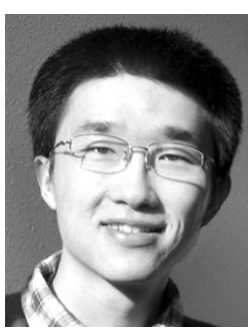

Hao Yang received Ph.D. degrees in automatic control from the Nanjing University of Aeronautics and Astronautics (NUAA) as well as Lille 1 University: Sciences and Technologies, France, both in 2009. In 2009, he joined the College of Automation Engineering of the NUAA, where he is currently an associate professor. He serves as an associate editor for Nonlinear Analysis: $H y$ brid Systems. His research interest includes stability and fault tolerant control of switched and hybrid systems, multi-agent systems, supervisory control and applications.

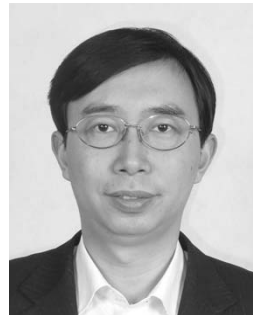

Bin Jiang obtained a Ph.D. in automatic control from Northeastern University, Shenyang, China, in 1995. Currently he is a full professor and a vice dean of the College of Automation Engineering at the Nanjing University of Aeronautics and Astronautics. He serves as an associate editor for IEEE Transactions on Control Systems Technology, International Journal of System Science, International Journal of Control, Automation and Systems, International Journal of Applied Mathematics and Computer Science, and others. His research interests include fault diagnosis and fault tolerant control and their applications.

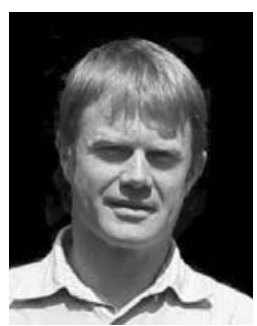

Vincent Cocquempot received the Ph.D. degree in automatic control from the Lille University of Sciences and Technologies, in 1993. He is currently a professor of automatic control and computer science at the University Technological Institute. He is the head of research in the LAGIS Laboratory, UMR CNRS 8219, Lille 1 University: Sciences and Technologies. His research interests include robust on-line fault diagnosis for uncertain dynamical nonlinear systems, fault detection and isolation, and fault tolerant control for hybrid dynamical systems.

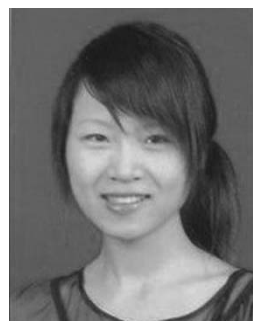

Lingli Lu is currently an M.Sc. student at the College of Automation Engineering, Nanjing University of Aeronautics and Astronautics, China. Her research interests include switching based fault diagnosis and fault tolerant control with application to unmanned aerial vehicles.

Received: 18 December 2010 Revised: 4 July 2011 